\title{
Powered addition applied to the fluidisation of a packed bed
}

\author{
P. D. de Wet $^{1}$, B. M. Halvorsen ${ }^{2,3}$ \& J. P. du Plessis ${ }^{1}$ \\ ${ }^{1}$ Department of Mathematical Sciences, Applied Mathematics Division, \\ Stellenbosch University, South Africa \\ ${ }^{2}$ Institute for Process, Energy and Environmental Technology, \\ Telemark University College, Norway \\ ${ }^{3}$ Telemark Technological R\&D Centre (Tel-Tek), Norway
}

\begin{abstract}
Upon analysis of a set of collected data of Newtonian flow through a porous medium, it is evident that asymptotes exist for some variable dependencies in the transition from packed to fluidised bed. The transition between such asymptotes is governed, amongst others, by parameters such as particle size, particle size distribution, superficial gas velocity and bed height. The powered addition to a power, $s$, of such asymptotes $f_{0}$ and $f_{\infty}$, leads to a single correlating equation that is applicable over the whole range of flow rates, namely $f^{s}=f_{0}^{s}+f_{\infty}^{s}$. This procedure circumvents the introduction of ad hoc curve fitting measures in the cross-over regions between the asymptotes and subsequent, unwanted jumps in piecewise fitted correlative equations for the dependent variable(s). The aforementioned method of powered addition is applied to the experimental data and the outcomes are discussed.
\end{abstract}

\section{Introduction}

A fluidised bed is formed when a fluid, usually a gas, is passed upwards through a bed of particles. The packed bed of particles, supported on some kind of distributor, is converted into an expanded, suspended bed and in the process takes on many liquid-like properties: the bed has zero angle of repose, pressure increases linearly with distance below the surface, wave motion is observed, denser objects sink and lighter objects may be floated on its surface, their movement almost unhindered [1]. 
A fluidised system has a number of highly useful properties that may be utilised in industrial applications. Although the mechanism may be both physical and chemical in nature, the dominating attribute utilised in a specific industrial process, will determine its application. A broad classification of fluid bed applications is given in [2], from which it is evident that fluidisation is an interdisciplinary field of inquiry. To optimally reap the benefits of this promising process there thus exists a particular need for the development of predictive models; holding challenges for both fundamental and applied research. The underlying motivation for this project is the aspiration towards establishing a sound modelling framework for analytical and computational predictive measures.

\section{Powered addition as curve-fitting technique}

It is common practice to represent the general trend in a set of collected data by drawing a line through the individual datum points on the plot. The better the predictive line on the graphical presentation corresponds to the physical reality, especially in the limits of the independent variable, the greater the trustworthiness of obtained results. The basic procedure of asymptotic matching by straightforward addition of the expressions for the asymptotic conditions is a method that has been in use for some time, especially in engineering practice. However, the article by Churchill and Usagi [3], which appeared in 1972, for the first time really formalised the use and accentuated the wide application possibilities of the method and variations thereof.

In many continuum processes the value of a sought after parameter, the dependent variable, is expressible as a function of certain known parameter(s), the independent variable(s), at low and high values. The latter may be regarded as asymptotic conditions of the dependent variable. Let a certain parameter, $f$, of such a process be described at low values of $x$ by

$$
f_{0} \equiv f\{x \rightarrow 0\}
$$

and the dependence at the upper extremal value of $x$ by

$$
f_{\infty} \equiv f\{x \rightarrow \infty\}
$$

The simple addition of two asymptotic solutions or approximations is often effected to obtain a single solution that holds over the entire range of the independent variable, i.e.

$$
f=f_{0}+f_{\infty}
$$

Equation (3) may now be considered as a matching curve connecting the two dependencies as it satisfies the asymptotic conditions and also provides values for $f$ at intermediate values of the independent variable, $x$. Frequently the values of the dependent variable at the transition between the asymptotic extremities do not lie exactly on this matching solution. Churchill and Usagi $[3,4]$ and Churchill $[5,6]$ demonstrated that the use of powered addition, as shown in equation (4), may lead 
to dramatic improvement in correlation with experimental data

$$
f^{s}=f_{0}^{s}+f_{\infty}^{s}
$$

By adjusting the value of the shifting exponent, $s$, the level of the solution may be modified so as to more closely trace the expected or empirical values, yielding better correspondence between predictive equation and experimental results.

\section{Experimental procedure}

Experimental data was obtained from measurements performed on laboratoryscale fluidized beds at Telemark University College, Porsgrunn, Norway. The bed was contained by a cylindrical perspex tube with an inner diameter of $72 \mathrm{~mm}$. Since the project was only concerned with the pressure drop across a specified section of the bed (i.e. between two pressure sensors), the porosity of the plate on which the bed was supported was irrelevant. It was only required not to allow particles to drop through into the antechamber and to function as a uniform gas distributor.

The beds were filled with glass powders consisting of spherical glass particles, with a density of $2485 \mathrm{~kg} / \mathrm{m}^{3}$ and available in three different diameter-ranges: $100 \mu \mathrm{m}-200 \mu \mathrm{m} ; 400 \mu \mathrm{m}-600 \mu \mathrm{m}$; and $750 \mu \mathrm{m}-1000 \mu \mathrm{m}$. These particles all fall into Group B and D according to the Geldart powder classification [7]. Since the particles are manufactured they were assumed, for the sake of simplicity, to be perfectly spherical in shape and thus have a Waddell sphericity factor, $\psi=1$ [8]. In all of the experiments performed the fluid used to fluidise the bed was air at ambient conditions, with a density of $1,2 \mathrm{~kg} / \mathrm{m}^{3}$ and viscosity of $1.78 \times$ $10^{-5} \mathrm{~N} \cdot \mathrm{s} / \mathrm{m}^{2}$.

By using different gas velocities and only one particle diameter-range or a mixture of the particle diameter-ranges, the parameters of the experiment could be varied.

A minimum of three runs were performed for each of the three different diameter-ranges mentioned above. A run consisted of a gradual increase until and beyond fluidisation, followed by a gradual and controlled decrease to zero fluid flow. It was noted that hysteresis only became apparent if the bed was allowed a period of rest between consecutive fluidisations. Consequently, runs for a particular diameter-range were conducted in succession allowing the obtained pressure-values to be averaged. It is to this averaged data that the curve-fitting technique discussed in this paper was applied.

\section{Curve fitting}

Three regimes are to be identified, namely the two regimes corresponding respectively to the physical conditions related to the two asymptotes $f_{0}$ and $f_{\infty}$ and the change-over regime connecting the two. This latter regime surrounds the critical point, $x_{c}$, where the asymptotes meet and is of particular interest in the present study. 


\subsection{The lower asymptote}

Before the onset of fluidisation, the bed may be regarded as a packed bed or porous medium consisting of spherical particles. To describe the pressure drop of Newtonian flow through such a structure, the Ergun equation has proven to be satisfactory in most applications as is evident from its extensive utilisation in chemical engineering. In their paper, Du Plessis and Woudberg [9] compare the RUC (representative unit cell) model to the Ergun equation for the description of Newtonian flow through a packed bed of uniformly sized spherical granules and find the agreement to be satisfactory. The choice, in this paper, of the RUC model to describe the lower asymptote is due to the fact that it is adaptable to different physical situations, whereas the Ergun equation is empirically based and will thus vary according to the situation to which it is applied. Furthermore it allows the usage of the average bed porosity and is applicable over both the entire porosity and laminar Reynolds number ranges.

In the original Ergun equation, which is already a special case of powered addition with shifter, $s=1$,

$$
\frac{\Delta p}{H}=A \frac{(1-\varepsilon)^{2}}{\varepsilon^{3}} \frac{\mu q}{D_{h}^{2}}+B \frac{1-\varepsilon}{\varepsilon^{3}} \frac{\rho_{f} q^{2}}{D_{h}},
$$

the values of coefficients $A$ and $B$ were acquired experimentally and are given as 150 and 1.75 respectively. Here $\Delta p$ denotes the finite pressure difference (measured in the stream-wise direction of fluid flow), $H$ the bed height, $\varepsilon$ the porosity or bed voidage, $\mu$ the fluid viscosity, $\rho_{f}$ the fluid density, $q$ the superficial velocity of the traversing fluid and $D_{h}$ the hydraulic diameter (which is equal to the diameter of the spherical particles).

The work of Du Plessis \& Woudberg allows one to purge equation (5) of its empirical elements. Pore-scale analysis of interstitial flow conditions lead to the following expression of coefficients

$$
A=\frac{25.4 \varepsilon^{3}}{(1-\varepsilon)^{2 / 3}\left(1-(1-\varepsilon)^{1 / 3}\right)\left(1-(1-\varepsilon)^{2 / 3}\right)^{2}},
$$

and

$$
B=\frac{\varepsilon^{2} c_{d}}{2\left(1-(1-\varepsilon)^{2 / 3}\right)^{2}} .
$$

They thus succeed in rewriting (5) such that it is independent of, and not limited by, the range of porosities used. Here the particle Reynolds number, $R e_{p}$, is defined as

$$
R e_{p} \equiv \frac{\rho_{f} q D_{h}}{\mu}
$$

and as in [9] the value of the form drag coefficient, $c_{d}$ in (7), was taken to be 1.9, presenting the most empirical aspect of the procedure. 


\subsection{The upper asymptote}

As noted by Geldart [8], the pressure drop across a fluidised bed, given by

$$
\Delta p=\frac{m_{0} g}{A_{c}}=\frac{\rho_{0} U_{0} g}{A_{c}}=\rho_{0} g H,
$$

is the only parameter that can be predicted with accuracy, since at all times during fluidisation the downward force, i.e. the weight of the bed, $m_{0} g$, is balanced by the upward force, $\Delta p A_{c}$. Division of equation (9) by the bed height, yields

$$
\frac{\Delta p}{H}=\frac{m_{0} g}{A_{c} H}=\frac{\rho_{0} U_{0} g}{A_{c} H}=\rho_{0} g
$$

which forms the upper limiting asymptote. Here $m_{0}$ denotes the bulk mass, $\rho_{0}$ the bulk density, $U_{0}$ the bulk volume, $g$ acceleration due to gravity (taken as $9.81 \mathrm{~m} / \mathrm{s}^{2}$ ) and $A_{c}$ the cross-sectional area of the bed.

\subsection{Powered addition of the asymptotes}

The original Ergun equation, (5), was obtained through simple addition of the Blake-Kozeny and Burke-Plummer equations, the former being a Darcy-type equation predominating in the regime where $R e_{p} \rightarrow 0$ and the latter dominating in the Forchheimer regime. If powered addition, as discussed in Section 2, is used to match the asymptotic conditions - i.e. equations (5) and (10) is combined - a single correlative measure,

$$
\frac{\Delta p}{H}=\left[\left(A \frac{(1-\varepsilon)^{2}}{\varepsilon^{3}} \frac{\mu q}{D_{h}^{2}}+B \frac{1-\varepsilon}{\varepsilon^{3}} \frac{\rho_{f} q^{2}}{D_{h}}\right)^{s}+\left(\rho_{0} g\right)^{s}\right]^{1 / s},
$$

is obtained for the pressure drop over the bed. Here coefficients $A$ and $B$ are as expressed in equations (6) and (7).

\subsection{Critical point and shifting-exponent}

The central or critical point, $x_{c}$, of the matching curve is the value of the independent variable at which the asymptotes meet. Since the asymptotes intersect here, the numerical value of their respective functional expressions must be equal, that is

$$
f_{0}\{x\}=f_{\infty}\{x\} .
$$

As both functions, $f_{0}$ and $f_{\infty}$, contribute equally to the added solution at this point, the resultant curve is most sensitive to variations in the value of the sifter, $s$, in the vicinity of $x_{c}$. 
Hence, to determine the value of the central point of the fluidised bed, we set

$$
A \frac{(1-\varepsilon)^{2}}{\varepsilon^{3}} \frac{\mu q}{D_{h}^{2}}+B \frac{1-\varepsilon}{\varepsilon^{3}} \frac{\rho_{f} q^{2}}{D_{h}}=\rho_{0} g,
$$

which yields a quadratic equation in $q$. Let $q_{c}$ be the value at which the asymptotes meet, i.e. the critical point. Solving $q=q_{c}$ in equation (13) yields

$$
q_{c}=\frac{A}{B} \frac{\mu}{2 \rho_{f} D}(1-\varepsilon)\left[-1 \pm \sqrt{\left(1+\frac{B}{A^{2}} \frac{\varepsilon^{3}}{(1-\varepsilon)^{3}} \frac{4 \rho_{0} \rho_{f} g D^{3}}{\mu^{2}}\right)}\right] .
$$

Since $\frac{B}{A^{2}} \frac{\varepsilon^{3}}{(1-\varepsilon)^{3}} \frac{4 \rho_{0} \rho_{f} g D^{3}}{\mu^{2}} \geq 0$ in equation (14) and $q_{c} \geq 0$, it follows that we may disregard the negative root. Substitution of this $q_{c}$ value into equation (11) will yield the function value at the intersection of the asymptotes. Determining the value of the shifting-exponent, $s$, we use the same argument as above in equation (12). Thus,

$$
f_{c}^{s}=f_{0}^{s}+f_{\infty}^{s}=2 f_{0}^{s}=2 f_{\infty}^{s},
$$

whence it follows that

$$
\left(\frac{f_{c}}{f_{\infty}}\right)^{s}=\left(\frac{f_{c}}{f_{0}}\right)^{s}=2 .
$$

The value of $s$ may now be determined straightforwardly from equation (16) as

$$
s=\frac{\ln 2}{\ln f_{c}-\ln f_{\infty}}=\frac{\ln 2}{\ln f_{c}-\ln f_{0}} .
$$

In performing an experiment, it is therefore advantageous to arrange the physical conditions in such a manner that the independent variable (here $q$ ) is in close vicinity of $x_{c}$ (here $q_{c}$ ). Whenever the experimental value of $f_{c}=f\left\{x_{c}\right\}$ is known, we proceed to determine the value of the shifter by equation (17).

Alternatively, visual inspection by trial and error adjustment of the correlation between the predictive curve and data points may lead to an assignment of a value to $s$. As noted by [3] the matched curve is relatively insensitive to variations in $s$; the required acuity being determined by considerations such as the process involved, tunability of other parameters and allowable error-margin.

\section{Correlation of experimental results}

The pressure drop was plotted against the superficial velocity for each of the diameter ranges. Figures 1 and 2 serve to illustrate the influence of the use of the minimum $\left(\mathrm{d}_{\min }\right)$, average $\left(\mathrm{d}_{\text {mean }}\right)$ and maximum $\left(\mathrm{d}_{\max }\right)$ particle diameters on the orientation of the lower asymptote. A similar plot may be drawn for particle diameter-range $400 \mu \mathrm{m}-600 \mu \mathrm{m}$. It is important to note that the asymptotes need not be straight lines; this is apparent in Figure 2 where the quadratic nature of the RUC model - equation (5) with coefficients $A$ and $B$ as expressed in equations (6) 


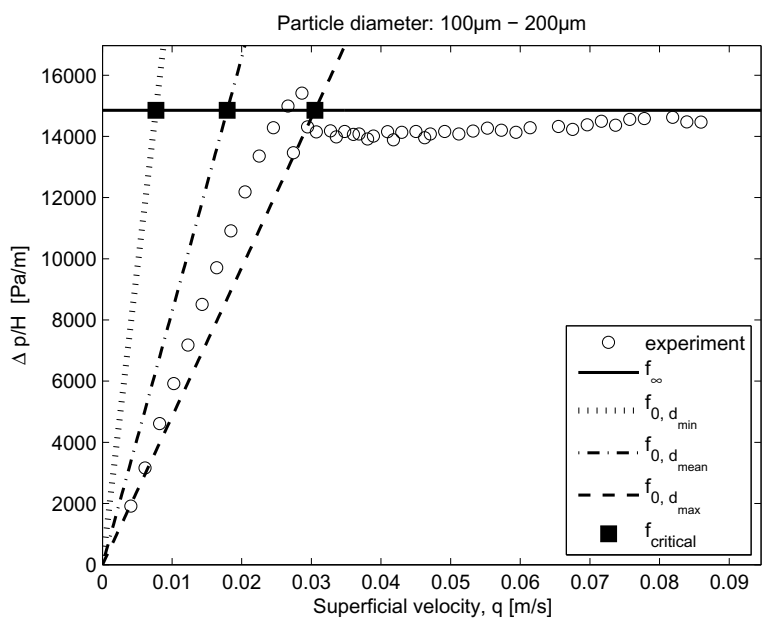

Figure 1: Particle diameter-range $100 \mu \mathrm{m}-200 \mu \mathrm{m}$. Influence of chosen particle diameter on the positioning of the lower asymptote, $f_{0}$, and the position of the critical point, $f_{c}$. The RUC model is used to describe the lower asymptote.

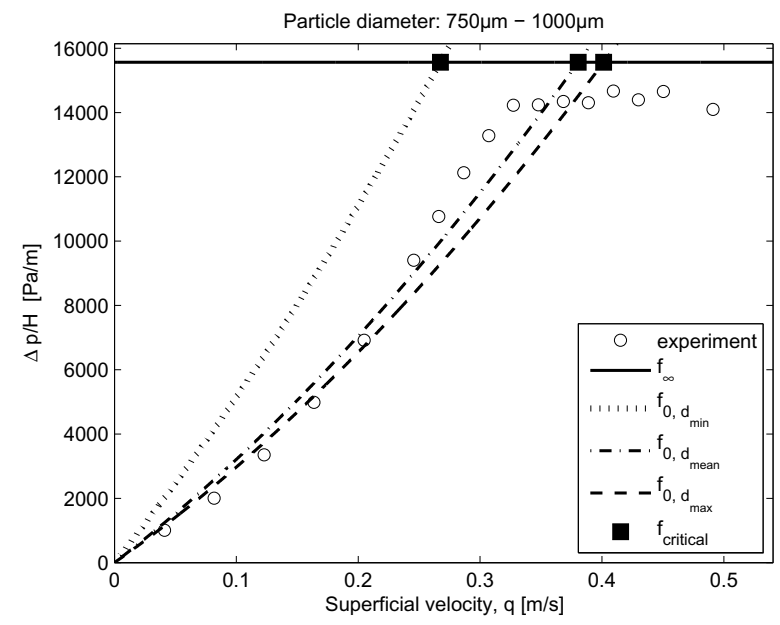

Figure 2: Particle diameter-range $750 \mu \mathrm{m}-1000 \mu \mathrm{m}$. The choice of particle diameter determines the orientation of the lower asymptote, $f_{0}$, and critical point, $f_{c}$. Note that the asymptotes need not be straight lines - slight curvature of $f_{0}$ due to the quadratic nature of equation (5) is evident. 


\begin{tabular}{|c|c|c|c|}
\hline & $100-200 \mu m$ & $400-600 \mu m$ & $750-1000 \mu m$ \\
\hline $\mathrm{d}_{\text {min }}$ & -0.593 & -0.825 & -1.878 \\
$\mathrm{~d}_{\text {mean }}$ & -2.248 & -3.155 & -8.193 \\
$\mathrm{~d}_{\text {max }}$ & -14.280 & -9.567 & -11.786 \\
\hline
\end{tabular}

Table 1: Calculated values of shifter-exponent, $s$, for the different particle diameter possibilities.

and (7) - starts to dominate due to an increase in the superficial velocity before the onset of fluidisation.

From these graphical results the particle-diameter yielding the best correlation with the experimental data was chosen to be used in the fitting of a predictive curve. In the case where the data points were not noticeably favouring a specific particle diameter, the experimental $q_{c}$-value closest matching the theoretically predicted value of the critical point, as expressed by equation (14), was used to determine an $s$-value. The corresponding curves were plotted and the best match was chosen by visual inspection. From equation (14) it is clear that the diameter of the particle impacts on the value of $q_{c}$, and thus on $f_{c}=f\left\{q_{c}\right\}$. The latter in turn has a direct influence on the value of $s$, as calculated by equation (17). Calculated values of $s$ are shown in Table 1.

For diameter-range $100 \mu \mathrm{m}-200 \mu \mathrm{m}$, shown in Figure 1, the experimental values lie between the average, $\mathrm{d}_{\text {mean }}$, and maximum, $\mathrm{d}_{\max }$, particle diameters. Curves for the matched solution were plotted for a particle diameter of both $153 \mu \mathrm{m}$ and $200 \mu \mathrm{m}$ - the former yielding results that closer match the trend of the data; illustrated by Figure 3 .

In the case of diameter-range $400 \mu \mathrm{m}-600 \mu \mathrm{m}$, the data points were distributed around the asymptote predicted by the upper limit of the range, $d_{\max }$. Use of a particle diameter of $600 \mu \mathrm{m}$ yields the best graphical results, as shown in Figure 4.

Examining diameter-range $750 \mu \mathrm{m}-1000 \mu \mathrm{m}$, it was once again difficult to discern the particle diameter to be used by merely regarding data point distribution about the asymptotes. Figure 2 shows experimental points crossing the asymptotes for both $\mathrm{d}_{\max }$ and $\mathrm{d}_{\text {mean }}$. Curves were plotted for both solutions; the average diameter of $960 \mu \mathrm{m}$ more closely followed the trend exhibited by the experimental data. The graphical result of a plot with this diameter is shown in Figure 5.

\section{Conclusion}

Although powered addition with a shifter, $s$, has not yet been proven to accurately describe the relative behaviour of the different parameters during a transfer process, it may be argued to be more appropriate, since the rate of change between the two asymptotes can be adjusted to fit experimental readings. For instance, the 




Figure 3: Curve-fitting by powered addition to experimental readings for particles in diameter-range $100 \mu \mathrm{m}-200 \mu \mathrm{m}$. The functional relation is given by equation (11). An $s$-value of -2.248 was used.



Figure 4: Powered addition-curve fitted to data for particles in diameter-range $400 \mu \mathrm{m}-600 \mu \mathrm{m}$. The functional relation is once again given by equation (11); $s$-value of -9.567 was used.

higher the value of the shifter-exponent, the more abrupt the changeover between the two asymptotic processes. Furthermore, irrespective of the rate at which the changeover occurs, it will be a smooth transition as is expected for the crossover from one continuum process to another. Thus, although primarily a curve-fitting 


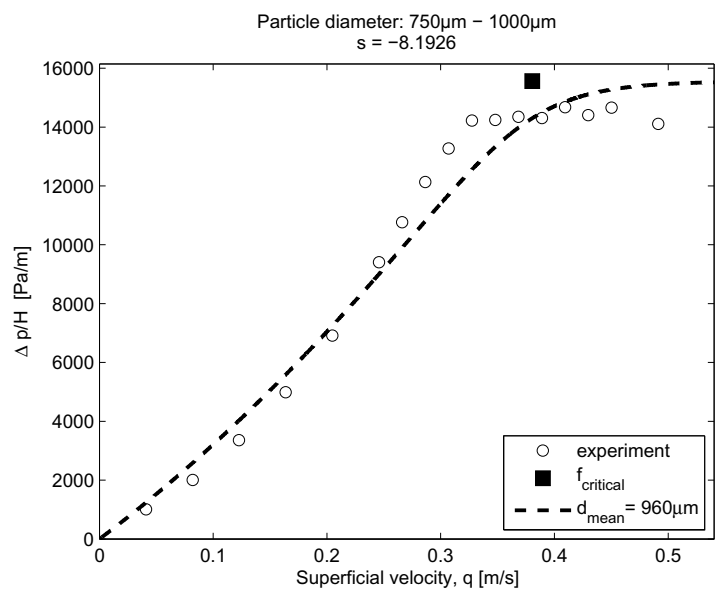

Figure 5: Curve-fitting by the method of powered addition of asymptotic solutions as applied to particles in diameter-range $750 \mu \mathrm{m}-1000 \mu \mathrm{m}$. Equation (11) represents the functional relation with an $s$-value of -8.193 having been used.

exercise, this procedure leads to better physical modelling since the only 'tuneable' parameter is the shifter-exponent, $s$. Adjusting its value does not change the value of the asymptotic conditions and leaves the double-asymptote character of the transfer process intact. The inherent simplicity of the method suggests that in such a curve-fitting exercise the greater deal of effort should be exerted in determination of the asymptotes, the value of the exponent, $s$, and possible relation of the latter to some quantifiable parameter.

\section{References}

[1] Davidson, J.F. \& Harrison, D., Fluidised Particles. Cambridge University Press: London, 1963.

[2] Geldart, D., Introduction (Chapter 1). Gas Fluidization Technology, ed. D. Geldart, John Wiley \& Sons, Inc.: Chichester, pp. 1 - 10, 1986.

[3] Churchill, S.W. \& Usagi, R., A general expression for the correlation of rates of transfer and other phenomena. American Institute of Chemical Engineers Journal, 18(6), pp. 1121 - 1128, 1972.

[4] Churchill, S.W. \& Usagi, R., A standardized procedure for the production of correlations in the form of a common empirical equation. Industrial and Engineering Chemistry, Fundamentals, 13(1), pp. 39 - 44, 1974.

[5] Churchill, S.W., The Interpretation and Use of Rate Data: The Rate Concept. McGraw-Hill: New York, 1974.

[6] Churchill, S.W., Derivation, selection, evaluation and use of asymptotes. Chemical Engineering and Technology, 11, pp. 63 - 72, 1988. 
[7] Geldart, D., Characterization of fluidized powders (Chapter 3). Gas Fluidization Technology, ed. D. Geldart, John Wiley \& Sons, Inc.: Chichester, pp. 33 - 54, 1986.

[8] Geldart, D., Single particles, fixed and quiescent beds (Chapter 2). Gas Fluidization Technology, ed. D. Geldart, John Wiley \& Sons, Inc.: Chichester, pp. $11-32,1986$.

[9] du Plessis, J.P. \& Woudberg, S., Pore-scale derivation of the Ergun equation to enhance its adaptability and generalization. Chemical Engineering Science, 63(1), pp. 2576 - 2586, 2008. 